\title{
Multistability Analysis and Function Projective Synchronization in Relay Coupled Oscillators
}

\author{
Ahmad Taher Azar $\mathbb{D},{ }^{1,2}$ Ngo Mouelas Adele, ${ }^{3}$ Kammogne Soup Tewa Alain $\left(\mathbb{D},{ }^{3}\right.$ \\ Romanic Kengne $(1),{ }^{3,4}$ and Fotsin Hilaire Bertrand $\mathbb{1}^{3}$ \\ ${ }^{1}$ Faculty of Computers and Information, Benha University, Benha, Egypt \\ ${ }^{2}$ School of Engineering and Applied Sciences, Nile University Campus, Sheikh Zayed District, Juhayna Square, \\ 6th of October City, Giza 12588, Egypt \\ ${ }^{3}$ Laboratoire de Matière Condensée d'Electronique et de Traitement du Signal, Faculty of Science, University of Dschang, \\ P.O. Box 67, Dschang, Cameroon \\ ${ }^{4}$ Research Group on Experimental and Applied Physics for Sustainable Development (EAPhySuD), P.O. Box 412, Dschang, Cameroon \\ Correspondence should be addressed to Ahmad Taher Azar; ahmad_t_azar@ieee.org
}

Received 22 July 2017; Revised 1 November 2017; Accepted 9 November 2017; Published 18 January 2018

Academic Editor: Sajad Jafari

Copyright (C) 2018 Ahmad Taher Azar et al. This is an open access article distributed under the Creative Commons Attribution License, which permits unrestricted use, distribution, and reproduction in any medium, provided the original work is properly cited.

\begin{abstract}
Regions of stability phases discovered in a general class of Genesio-Tesi chaotic oscillators are proposed. In a relatively large region of two-parameter space, the system has coexisting point attractors and limit cycles. The variation of two parameters is used to characterize the multistability by plotting the isospike diagrams for two nonsymmetric initial conditions. The parameters window in which the jerk system exhibits the unusual and striking feature of multiple attractors (e.g., coexistence of six disconnected periodic chaotic attractors and three-point attraction) is investigated. The second aspect of this study presents the synchronization of systems that act as mediators between two dynamical units that, in turn, show function projective synchronization (FPS) with each other. These are the so-called relay systems. In a wide range of operating parameters; this setup leads to synchronization between the outer circuits, while the relaying element remains unsynchronized. The results show that the coupled systems can achieve function projective synchronization in a determined time despite the unpredictability of the scaling function. In the coupling path, the outer dynamical systems show finite-time synchronization of their outputs, that is, displaying the same dynamics at exactly the same moment. Further, this effect is rather general and it has a wide range of applications where sustained oscillations should be retained for proper functioning of the systems.
\end{abstract}

\section{Introduction}

Multistability, meaning the coexistence of many different kinds of attractors, is an intrinsic property of many nonlinear dynamical systems and has become very important research topic and received much attention recently $[1,2]$. Multistability poses a threat for engineering systems because the system may unpredictably switch into an undesirable state. Multistability exhibits a rich diversity of stable states of a nonlinear dynamical system and makes the system offer a great flexibility. Particularly, when the number of coexisting attractors generating from a dynamical system tends to be infinite, the coexistence of many attractors depending on the initial condition of a certain state variable is alleged to be extreme multistability [3]. The occurrence of multiple attractors, which implies multiple stability and thus hysteretic dynamics, is one of the most important phenomena encountered in nonlinear dynamical systems. Such type of behavior has been reported in a wide range of systems including electronic circuits [4], laser [5], biological systems [6], Lorenz system [7], Josephson junction [8], and chemical reactions [9]. Multiple attractor bifurcations are said to occur when multiple coexisting attractors are simultaneously created at a bifurcation point [10]. It has been shown earlier that in some cases border collision bifurcations may lead to multiple attractor bifurcations [11]. 
More recently, Bao and collaborators [12] developed hidden extreme multistability in memristive hyperchaotic system. In that paper, they established a novel memristive hyperchaotic system with no equilibrium based on the newly proposed circuit realization scheme and investigated the phenomenon of extreme multistability with hidden oscillation that reveals the coexistence of infinitely many hidden attractors in the proposed memristive hyperchaotic system. Kengne et al. [13] presented the basic dynamical properties of a simple autonomous jerk system including equilibria and stability, phase portraits, frequency spectra, bifurcation diagrams, and Lyapunov exponent plots. It is shown that the onset of chaos is achieved via the classical period-doubling and symmetryrestoring crisis scenarios. One of the key contributions presented in their work was that the jerk system experiences the striking feature of multiple attractors (e.g., coexistence of four disconnected periodic and chaotic attractors) [13, 14]. It is important to note that the results obtained revealed that there are some unexplored parameters' regions of this circuit where four disconnected nonstatic attractors coexist.

The interaction of two nonlinear systems via a third parameter-matched circuit typically leads to a variety of significant behaviors, among which the most intriguing is probably synchronization (known usually as relay synchronization), that is, the coordination of a particular dynamical property of their motion [15]. The interaction between two chaotic systems has been deeply studied during the past decade, focusing on the ability of synchronization even in the presence of noise or delay. In [16], Wagemakers et al. examined the robustness of isochronous synchronization in simple arrays of bidirectionally coupled systems. The results of the study showed experimentally that the relaying unit does not need to be identical to the outer systems which are the ones to be synchronized. Sharma et al. in [17] proposed the dynamics of nonlinear oscillators indirectly coupled through a dynamical environment. The results of the study showed that this form of indirect coupling leads to synchronization and phase-flip transition in periodic as well as chaotic regime of oscillators. The phase-flip transition in the case of relay coupled system was investigated by Sharma et al. in [18]. In that paper, the authors show that, in the absence of time delay, relay coupling through conjugate variables has the same effect as when the interactions involve a time delay. However, this phase-flip transition does not occur abruptly at a certain critical value of the coupling parameter. Relay synchronization (RS) has been used with electronic circuits, as a technique for transmitting and recovering encrypted messages, which can be sent bidirectionally and simultaneously [19]. Apart from its technological applications, RS has also been proposed as a possible mechanism at the basis of isochronous synchronization between distant areas of the brain [20]. Nana and Woafo proposed a theoretical and experimental synchronization of three oscillators coupled as emitter-relay-receiver system [21]. They proposed an experimental setup and showed that it is impossible to achieve a zero synchronization error due to the tolerances of the electrical components. Some demonstrations of chaotic masking of communication as well as selected secure communication lines were observed. Gutiérrez et al. in [22] showed that a generalized synchronization (GS) in relay systems with instantaneous coupling could be obtained. The authors proved the existence of GS in unidirectional coupled units (drive system $\rightarrow$ response system) by checking the ability of the response system to react identically to different initial conditions of the same driver system, which can be quantified by evaluating the mutual false nearest neighbors or by measuring the conditional Lyapunov exponents. Despite such evidence of RS, there are still open questions of a fundamental nature. The main issue is to characterize properly the relationship established in RS between the dynamics of the relay system and that of the synchronized systems. From the previously mentioned references, the literature needs a strict analysis of the performance of the RS using a scaling function. Besides, the projective synchronization (PS) has been used in the research of secure communication because of the unpredictability of the scaling function which may be a useful element [23]. So the development of the function projective synchronization in relay systems is important challenging research point. This motivates the present study.

The aim of this work is to make some dynamical analysis details of complex systems that can exhibit many major features of the regular and chaotic motion which allows a better understanding of its behavior and providing a generic route of function synchronization in relay coupled jerk oscillators.

The remainder of the paper is organized as follows. In Section 2, the nonlinear system is presented and the dynamical behaviors of the circuit are identified with the help of a numerical two-parameter Lyapunov exponent diagram. The finite-time synchronization issue is formulated in Section 3 in which synchronization and numerical simulations are presented. Finally, conclusions and remarks are given in Section 4.

\section{The Model and Its Behavior}

2.1. Preliminaries. We consider the following chaotic system:

$$
\begin{gathered}
\dot{z}_{i}(t)=a_{i} z_{i+1}(t), \quad i=1,2, \ldots, n-1 \\
\vdots \\
\dot{z}_{n}(t)=f(z(t)),
\end{gathered}
$$

where $z(t)=\left(z_{1}(t), z_{2}(t), \ldots, z_{n}(t)\right)^{T}$ is the system state vector, $a_{i}$ the constants, and $f(z(t))$ the nonlinear smooth function. Actually, through topological transformation, many existing chaotic systems, such as Chen systems, Lorenz systems, Lu systems, can be transformed as in the form of system (1). More recently, a growing interest is in the analysis of the LEs on Lyapunov diagrams, where we associate colors for the largest and the second largest exponent varying simultaneously two system's parameters [24, 25]

Remark 1. In real world, the order of chaotic system (1) usually will not go beyond fourth order. Therefore, the subscripts is less than or equal to 4 (i.e., $i \leq 4$ ).

If we set $i=3$, the general class of Genesio-Tesi system is obtained. This system is one of paradigms of chaos since it captures many features of chaotic systems. It includes a 


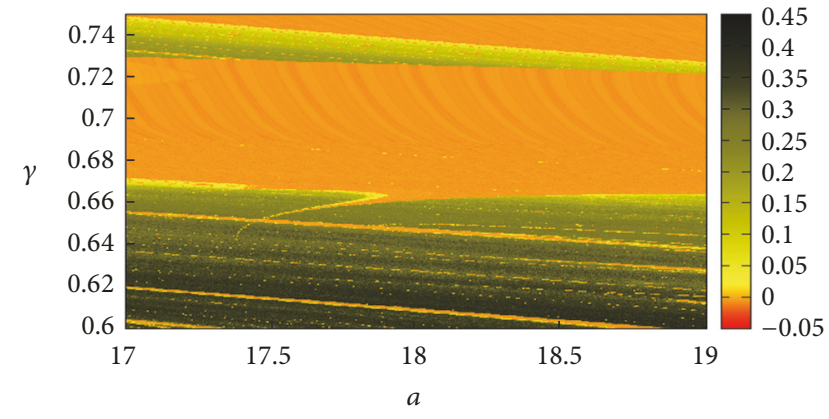

(a)

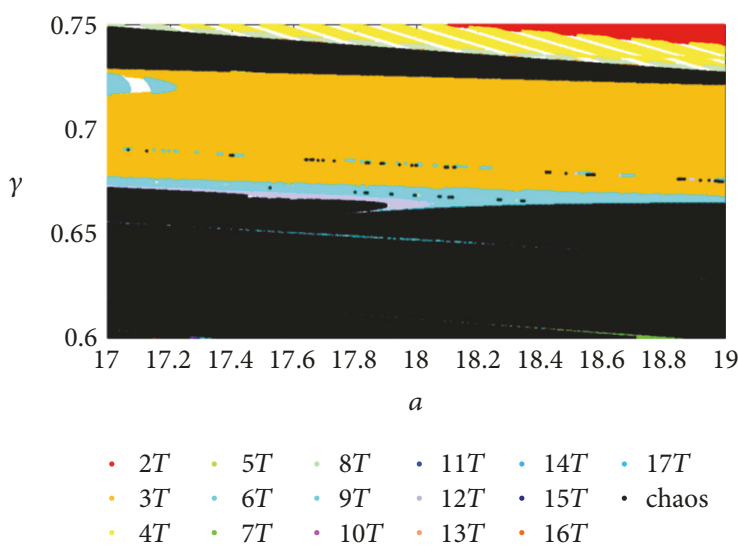

(b)

Figure 1: Two complementary ways of characterizing the stability of the MO5 circuit in the plan (a, $\gamma$ ): (a) Lyapunov stability diagram, where the orange shadings mark periodic oscillations and yellow and black denote the chaotic behaviors; (b) isospike diagrams displaying the number of peaks in one period of $x(t)$. These two figures are plotted for the initial conditions $(0.5,0,0)$. Regularity, multiperiodicity and "chaos" are represented, respectively, in (b). Each color shows the number of peaks in one period $T$ of $x(t)$.

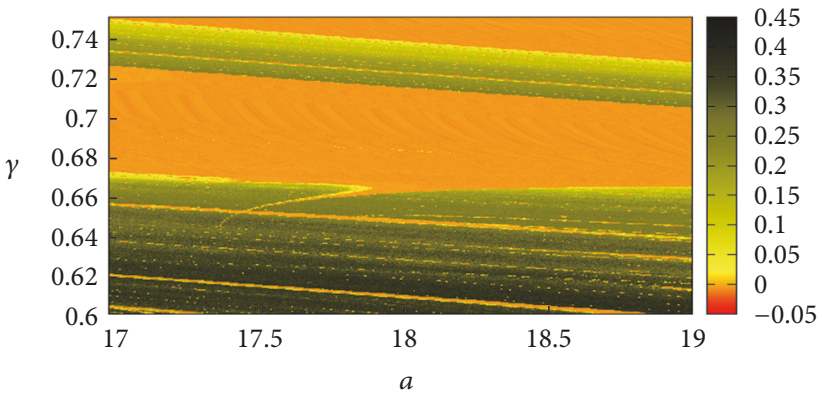

(a)

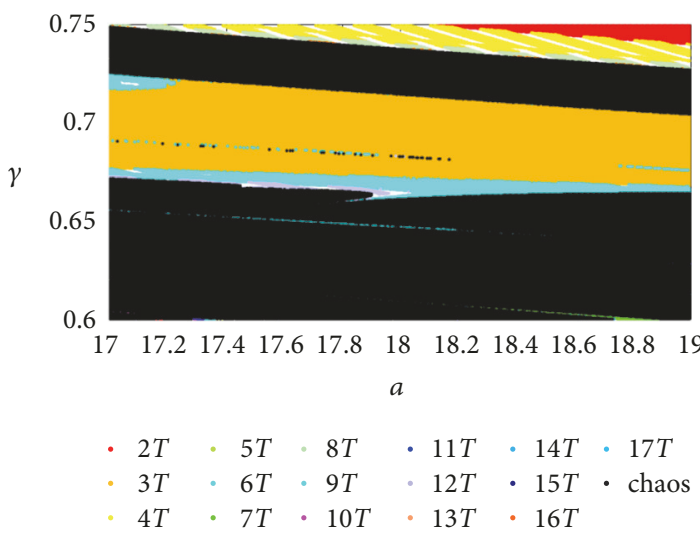

(b)

Figure 2: In (a) Lyapunov diagram for the $(a, \gamma)$ plane and in (b) isospike diagram for the same range of parameters as in (a) with the initial conditions set at $(0,0.5,0.5)$. These figures have been plotted with the same rank as the parameters in Figure 1.

simple square part and three simple ordinary differential equations that depend on three positive real parameters. Let us consider for this study the simple autonomous jerk system with multiple attractors presented recently by Kengne et al. and described by the following dynamics equations $[13,14]$ :

$$
\begin{aligned}
& \dot{x}_{1}=x_{2}, \\
& \dot{x}_{2}=a x_{3}, \\
& \dot{x}_{3}=-\gamma x_{2}-x_{3}+p\left(x_{1}\right),
\end{aligned}
$$

where $a$ and $\gamma$ are the positive constants and $p\left(x_{1}\right)$ the polynomial smooth function. For instance, the system is chaotic for the parameters $a=10, \gamma=0.725, p\left(x_{1}\right)=x_{1}-x_{1}^{3}$. Equation (2) represents a reliable and palpable resource for generating a wide variety of nonlinear phenomena including the multiple stability behavior. This system is capable of displaying many disconnected attractors (for some suitable sets of parameters) depending solely on the choice of initial conditions [13]. The following section underlines some unexplored parameter's regions of systems proposed in (2) which shows that many attractors coexist.

2.2. Stability Analysis of the Attractors. This section presents in two complementary ways (described below) phase diagrams characterizing the far-reaching regular organization induced by the set of stable oscillations of the circuit. Although obtained using two very distinct algorithms, the boundaries between chaotic and periodic regions match perfectly by plotting on a fine parameter grid the largest nonzero Lyapunov exponent. Such exponents are familiar indicators that allow one to discriminate chaos (positive exponents) from periodic oscillations (negative exponents). Figures 1 and 2 depict the behavior of MO5 oscillator in the plane $(a, \gamma)$ for a mesh of $750 \times 750$ parameters points. The results are obtained by using the standard fourth-order Runge-Kutta algorithm with fixed time step $h=5 \times 10^{-3}$. Figures 1 and 2 are obtained by adopting the initial values 
as $(0.5,0,0)$ and $(0,0.5,0.5)$, respectively. As usual the first $5 \times 10^{5}$ integration step disregarded as a transient time is considered to approach the attractor. The discrimination of the solutions and the account of the number of peaks within a period of $x(t)$ are detailed in [25]. Figures 1(a) and 2(a) display the Lyapunov stability diagram, obtained by plotting in two dimensions (in the plane $(a, \gamma)$ ) the largest nonzero Lyapunov exponent for the same parameters. The initial conditions are adopted as $(0.5,0,0)$ and $(0,0.5,0.5)$, respectively. The orange shadings mark periodic oscillations (negative exponents); the yellow and black colors denote the chaotic behaviors (positive exponent). It is worth noting that the diagrams plotted for the same values of parameters and the different initial conditions should be identical in the case where the circuit depicts no multistability fashion. This aspect is not observed in these two figures. The Lyapunov exponent points out this difference. This method is limited because it can only bring out the regions of coexistence between chaos and regularity, when plotting the isospike diagrams to complete the analysis. Figures 1(b) and 1(c) display the isospike diagrams in the plane $(a, \gamma)$ for the same values of the parameters for the following initial conditions fixed as $(0.5,0,0)$ and $(0,0.5,0.5)$, respectively. We use a palette of 17 colors to represent the number of spikes contained in one period of the oscillate state $x(t)$ as indicated by the color dots. Within the parameter range chosen, we obtain $2,3,4,5,6,7,8,9,10,11,12,13$, $14,15,16$, and 17 spikes in a single period $T$ of $x(t)$. The black color denotes the chaotic behavior. These two figures are used to study the 2D multistability in MO5 oscillators. They consistency shows all coexistences between the regular and nonregular oscillations.

2.3. Occurrence of Multiple Attractors. Most systems have only one attractor or one single type of attractor. Others may have two different types of coexisting attractors, most likely strange attractors and periodic cycles. It is interesting and striking to see that the simple system reported here has all three different common types of attractors coexisting side by side. Figure 2(a) presents the two-parameter Lyapunov exponent diagrams in the plane for the following initial conditions $(0,0,0.5)$. The isospike is presented in Figure 2(b). The same features observed in Figure 1(b) are also observed with additional remarks. For instance, in Figure 1(b) we observe two black bands indicating the chaotic domains. One of these bands corresponds to the high values of $\gamma$ which borders two distinct periodic structures; the structure of period- 3 and period- 6 and decreases gradually when the parameter $a$ is monitored, whereas in Figure 2(b) the same band for the different conditions is encircled by two distinct periodic structures, namely, period- 3 and period- 6 in the three regions; the band here remains solid in its evolution showing the stability of the chaotic attractors. The difference between Figures 1(b) and 2(b) is a fundamental concept resulting from the multistability of attractors. In this work, we presented the multistability zones for the same ranges of variation of the plane $(a, \gamma)$.

Previous work [13] reports in some way a detailed dynamical analysis of the system proposed in this work and discovered that the system is capable of displaying four
TABLE 1: Coexistence of multiple solutions for suitable values of parameters $(a, \gamma)$.

\begin{tabular}{lcccc}
\hline $\begin{array}{ccc}\text { a } \\
\text { 17.63 }\end{array}$ & $\gamma$ & $\begin{array}{c}\text { Initial } \\
\text { conditions }\end{array}$ & $\begin{array}{c}\text { Symmetric coexistence of two } \\
\text { attractors }\end{array}$ \\
& 0.6714 & $( \pm 0.5,0,0)$ & Period-6 & Figure 6(a1) \\
& & $(0, \pm 0.5, \pm 0.5)$ & Period-4 & Figure 6(a2) \\
18.16 & 0.7206 & $( \pm 0.5,0,0)$ & Period-5 & Figure 6(b1) \\
& & $(0, \pm 0.5, \pm 0.5)$ & Period-3 & Figure 6(b2) \\
18.22 & 0.6816 & $( \pm 0.5,0,0)$ & Period-3 & Figure 6(c1) \\
& & $(0, \pm 0.5, \pm 0.5)$ & Period-6 & Figure 6(c2) \\
17.37 & 0.7401 & $( \pm 0.5,0,0)$ & Chaos & Figure 6(d1) \\
& & $(0, \pm 0.5, \pm 0.5)$ & Period-6 & Figure 6(d2) \\
18.03 & 0.7191 & $( \pm 0.5,0,0)$ & Chaos & Figure 6(e1) \\
& & $(0, \pm 0.5, \pm 0.5)$ & Period-3 & Figure 6(e2) \\
\hline
\end{tabular}

disconnected attractors (for some suitable sets of parameters) depending solely on the choice of initial conditions and six disconnected ones using the perturbations method [13-15]. There is a relatively large parameters space of coexisting attractors located on the top of Figure 2(a) where a symmetric pair of point attractors coexists with other attractors. Our analysis shows an additional feature of the coexistence of multiple attractors involving a pair of asymmetric chaotic attractors with a pair of asymmetric periodic ones. Six disconnected attractors with three coexisting equilibrium points attractors are presented instead of four reported in [13] previously mentioned. The three coexisting points obtained, respectively, with the following initial conditions $(-1,0,0)(0,0,0)(1,0,0)$ are an interesting feature of this circuit. We recall that Sprott reported two coexisting equilibriums for a dynamical system with four quadratic nonlinearities to display a butterfly strange attractor. As shown in Figures 3 and 4, the main coexisting regime is a symmetric pair of point attractors coexisting with a symmetric periodic cycle. However, there are several other coexisting regimes as shown in Table 1. Out of well-known dynamics of the chaotic systems encountered in the literature, we present another interesting feature at period-12, two symmetric attractors, conjugated in the phase plan with a nonsymmetric initial condition. Figure 5 displays these dynamics.

The basins of attraction of the different attracting sets provide more information about the coexisting attractors, which are defined as the set of initial conditions whose trajectories converge to the respective attractor. For the following values of the parameters $a=17.37$ and $\gamma=0.7401$ where a symmetric pair of point attractors coexists with a symmetric pair of limit cycles in Figure 4(d), the basins in the $x_{2}=0$ (the second coordinate of system (1)) plane are shown in Figure 7. The basins of the two point attractors are indicated by yellow, red, and black, respectively, for the pair of period- 3 and period- 6 and the pair of chaos. The blue part represents the unbounded solution. The basins have the expected symmetry about the $y$-axis and a fractal boundary. 


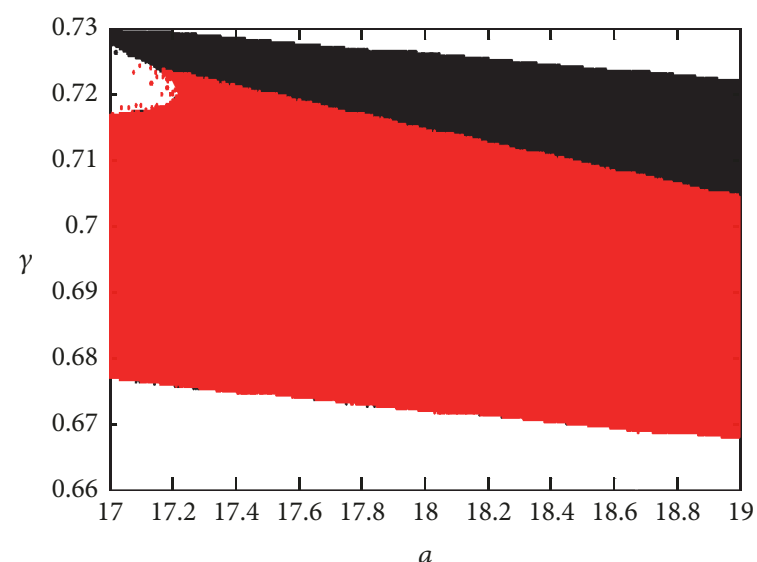

(a)

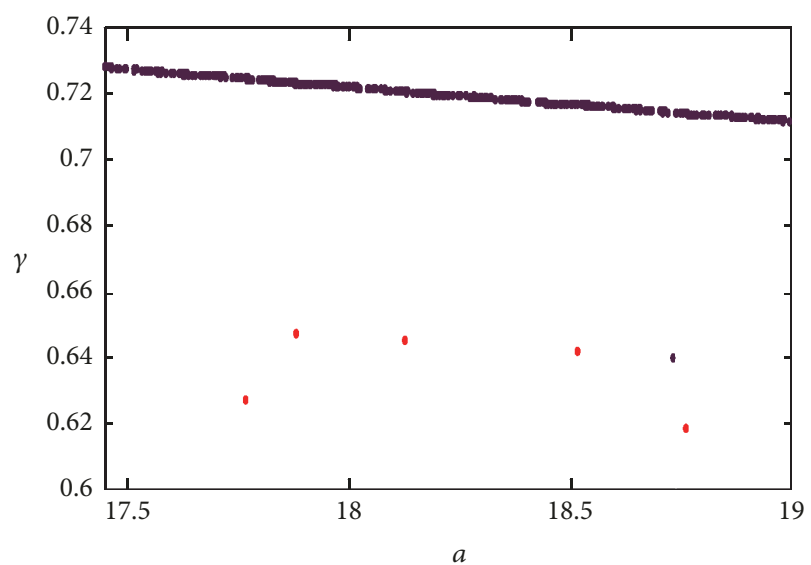

(b)

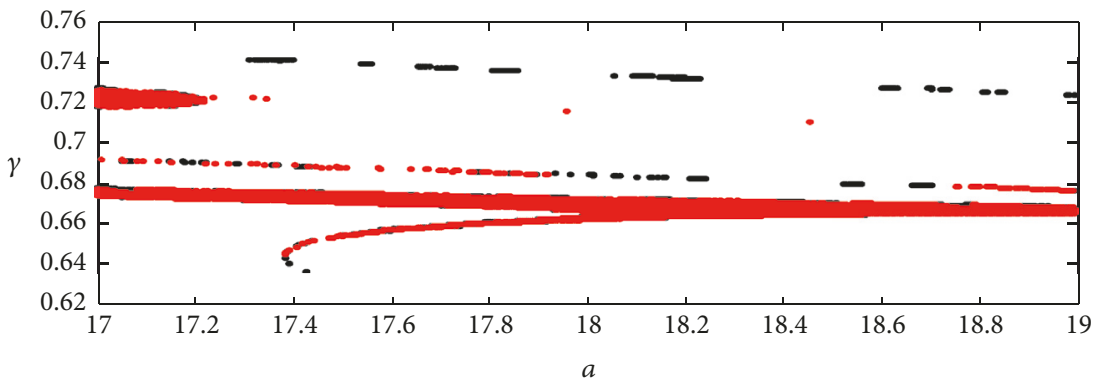

(c)

FIGURE 3: Isospike diagram in the $(a, \gamma)$ panel, the red color codifies the zones with initial conditions $(0.5,0,0)$ and the blue color codifies the zones corresponding to initial conditions $(0,0.5,0.5)$ : (a) 3 peaks in one period of $x(t)$; (b) 5 peaks in one period of; (c) 6 peaks in one period of. The region where the black color is visible is the region of $2 \mathrm{D}$ multistability.

\section{Function Projective Synchronization in Relay Coupled Oscillators}

3.1. Problem Formulation. Now, let us present a theory of the function projective synchronization in relay coupled systems. Our scheme is given as follows: The outer systems noted $X$ and $Y$ are the systems to synchronize while the relay unit is noted by $(R)$. From this consideration, the outer systems are described as follows:

$$
\begin{gathered}
\dot{z}_{1}(t)=a_{1} z_{2}(t)-\xi\left(z_{1}(t), \rho_{1}(t), m(t)\right), \\
\dot{z}_{i}(t)=a_{i} z_{i+1}(t), \quad i=2, \ldots, n-1 \\
\vdots \\
\dot{z}_{n}(t)=f(z(t)),
\end{gathered}
$$

where $z(t)$ represents $X(t)=\left(x_{1}(t), x_{2}(t), \ldots, x_{n}(t)\right)^{T}$ or $Y(t)=\left(y_{1}(t), y_{2}(t), \ldots, y_{n}(t)\right)^{T} . \xi\left(z_{1}(t), \rho_{1}(t), m(t)\right)$ is the coupling strength function.

The relay system can take the following form:

$$
\begin{aligned}
& \dot{\rho}_{i}(t)=a_{i} \rho_{i+1}(t)+u(t), \quad i=1,2, \ldots, n-1, \\
& \dot{\rho}_{n}(t)=f(\rho(t))+u_{n}(t),
\end{aligned}
$$

where $u(t)=\left[u_{1}(t), u_{2}(t), \ldots, u_{n}(t)\right]^{T}$ is the controller to be determined.
Definition 2. If there exists a scaling function $m(t)$ satisfying

$$
\lim _{t \rightarrow t_{0}}\|e(t, m(t))\|=0 \quad \forall e\left(t_{0}, m\left(t_{0}\right)\right) \in R^{n}
$$

then systems (3) and (4) achieve projective synchronization.

Remark 3. Chaos synchronization schemes such as complete synchronization and antisynchronization are special cases of function projective synchronization when $m(t)=1$ and $m(t)=-1$, respectively.

Taking into account the synchronization between two chaotic systems, take the drive relay as follows:

$$
\begin{gathered}
\dot{\rho}_{1}=a_{1} \rho_{2}+u_{1}, \\
\dot{\rho}_{2}=a_{2} \rho_{3}+u_{2} \\
\vdots \\
\dot{\rho}_{n}=\gamma_{2} \rho_{2}+\gamma_{3} \rho_{3}+\cdots+\gamma_{n} \rho_{n}+p\left(\rho_{1}\right)+u_{n} .
\end{gathered}
$$

Let us define the synchronization errors between our systems as follows:

$$
\begin{gathered}
e_{1}=x_{1}+y_{1}-2 m(t) \rho_{1}, \\
e_{2}=x_{2}+y_{2}-2 \rho_{2} \\
\vdots \\
e_{n}=x_{n}+y_{n}-2 \rho_{n} .
\end{gathered}
$$




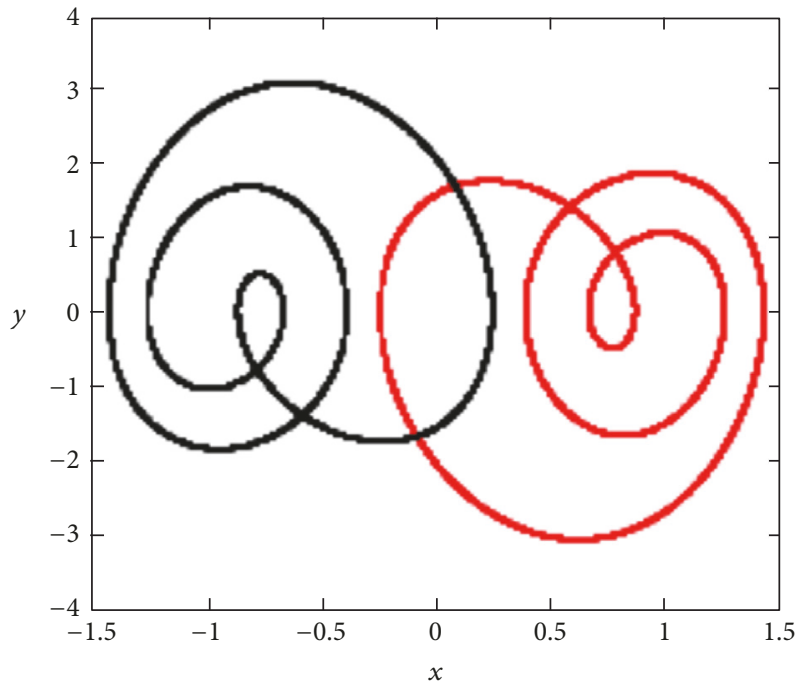

(a)

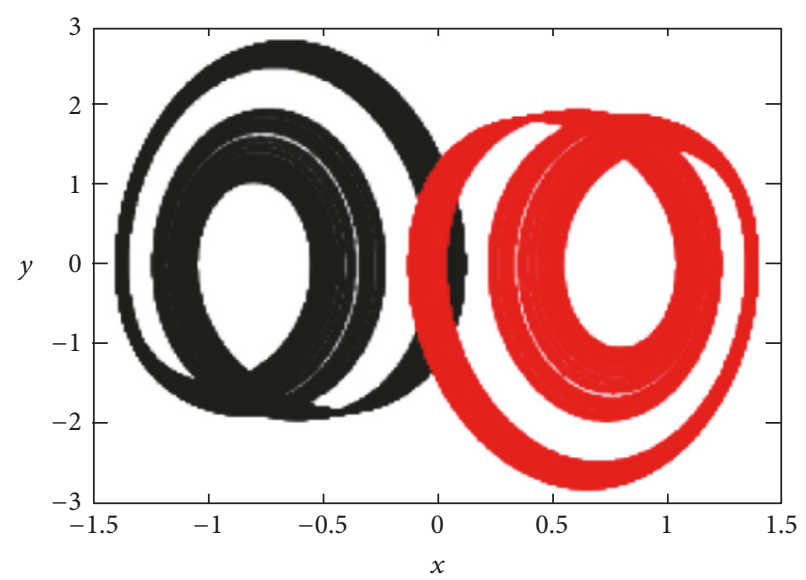

(c)

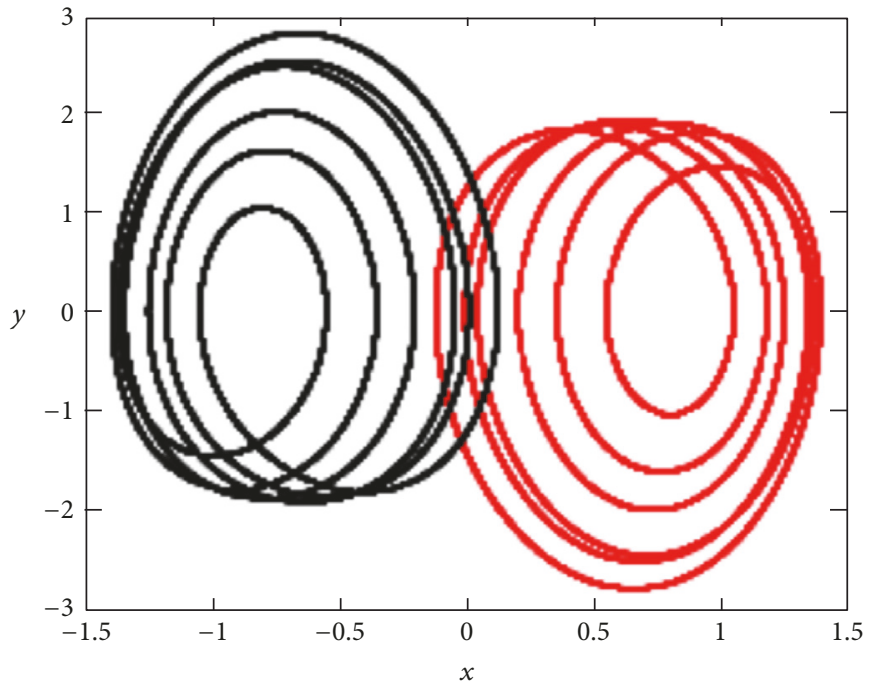

(b)

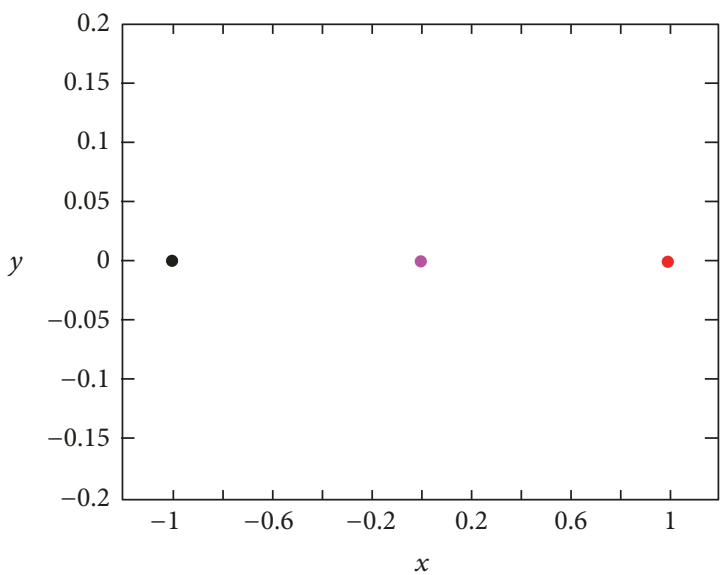

(d)

FIGURE 4: Phase portrait of coexisting attractors in the $x-y$ plane: $a=17.37, \gamma=0.7401$, with the following initial conditions: (a) ( $\pm 1,0$, \pm 0.01 ); (b) $( \pm 0.4,0, \mp 0.6)$; (c) $( \pm 0.58,0, \mp 0.6)$; (d) $(1,0,0)$ for black attraction point, $(0,0,0)$ for magenta attraction point, and $(-1,0,0)$ for red attraction point (red and black with symmetric initial conditions).

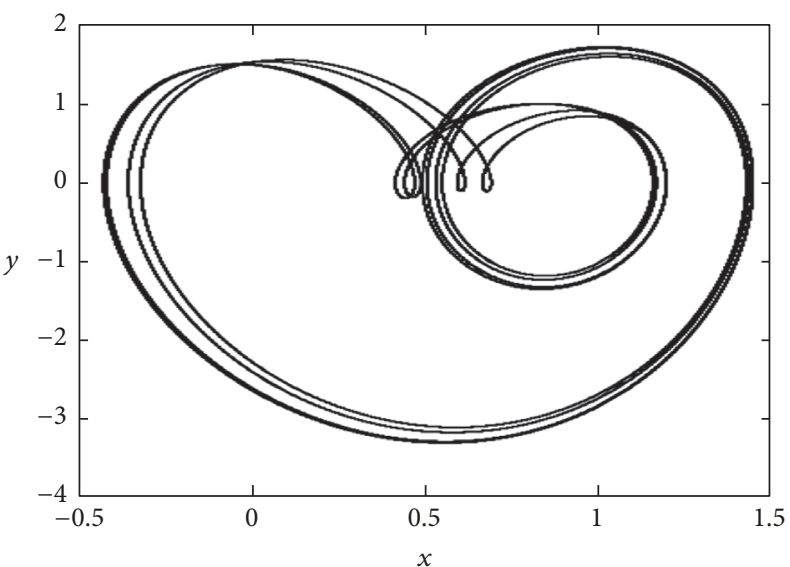

(a)

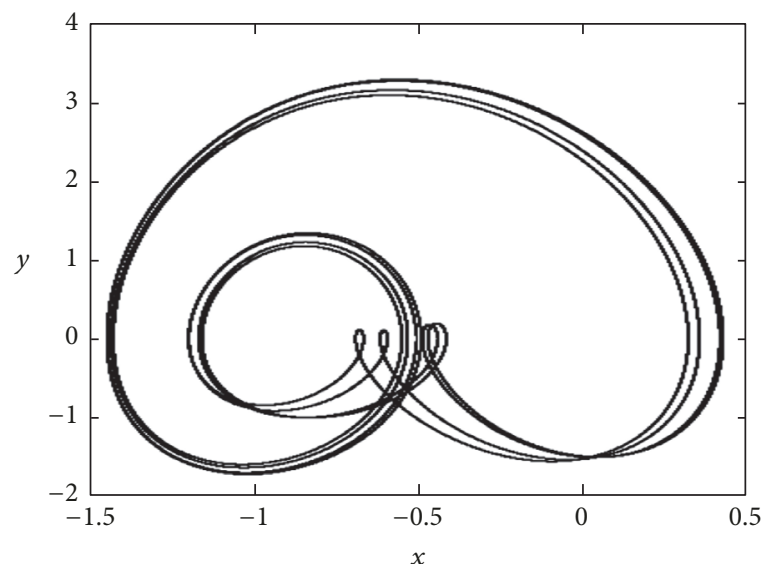

(b)

Figure 5: (a) Period-12 for $a=17.7$ and $\gamma=0.6678$ with initial conditions $(0.5,0,0)$. (b) Period-12 for $a=17.7$ and $\gamma=0.6678$ with initial conditions $(0,0.5,0.5)$. 


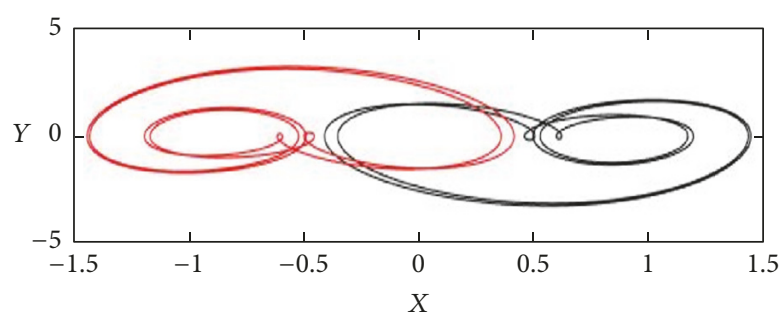

$\left(a_{1}\right)$

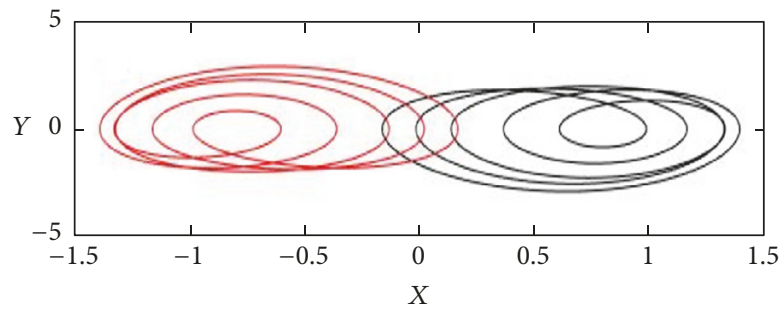

$\left(b_{1}\right)$

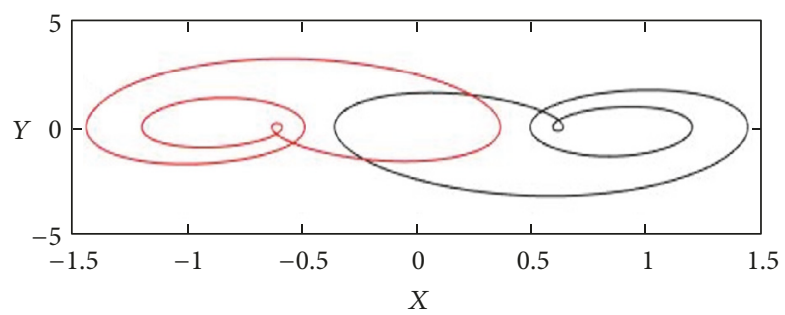

$\left(c_{1}\right)$

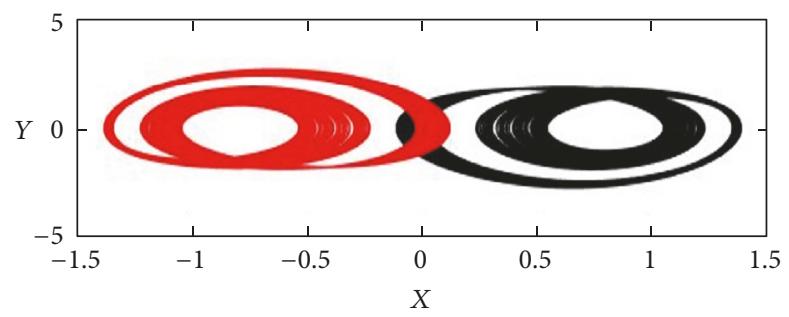

$\left(\mathrm{d}_{1}\right)$

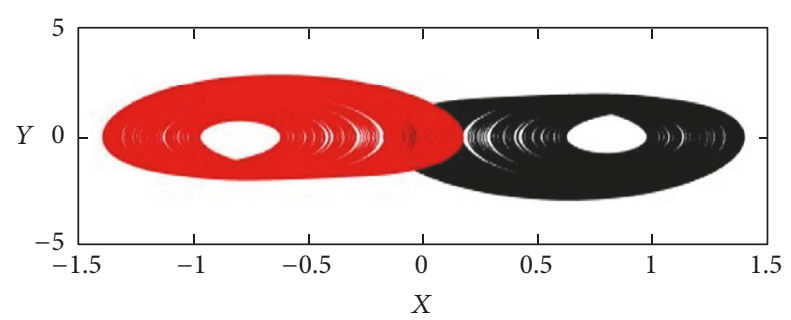

$\left(\mathrm{e}_{1}\right)$

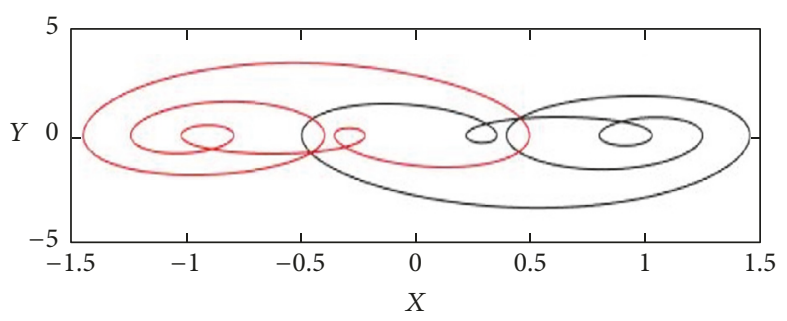

$\left(a_{2}\right)$

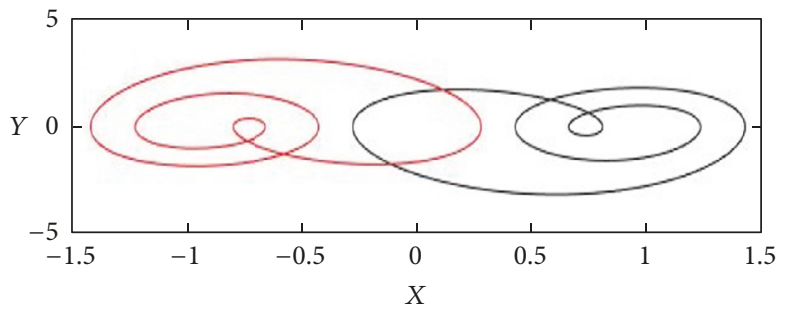

$\left(b_{2}\right)$

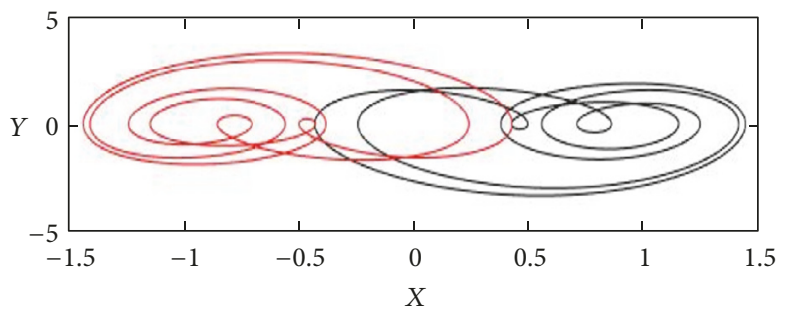

$\left(c_{2}\right)$

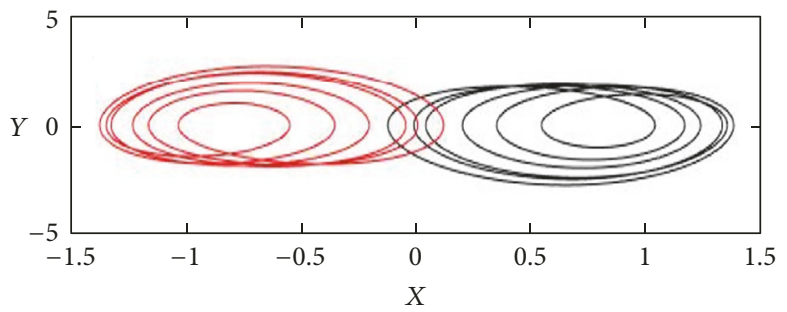

$\left(d_{2}\right)$

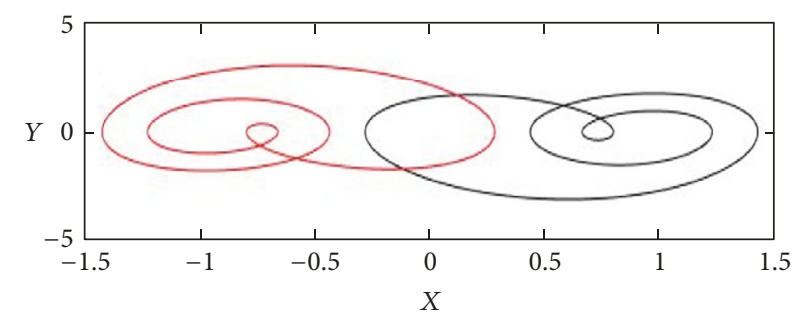

$\left(e_{2}\right)$

FIGURE 6: Coexistence of four different attractors (a pair of period-3, period-5, and period-6 and a pair of chaotic attractors) for (a), (b), (c), (d), and (e), respectively, represented in Table 1 . Notice that attractors are symmetric in pairs.

Considering (7), the dynamics of the errors become

$$
\begin{gathered}
\dot{e}_{1}=\dot{x}_{1}+\dot{y}_{1}-2 m(t) \dot{\rho}_{1}-2 \dot{m}(t) \rho_{1}, \\
\dot{e}_{2}=\dot{x}_{2}+\dot{y}_{2}-2 \dot{\rho}_{2} \\
\vdots \\
\dot{e}_{n}=\dot{x}_{n}+\dot{y}_{n}-2 \dot{\rho}_{n} .
\end{gathered}
$$

From these equations, we obtain the following error dynamics:

$$
\begin{aligned}
\dot{e}_{1} & =-k e_{1}+a_{1}\left(x_{2}+y_{2}\right)-2 a_{1} m(t) \rho_{2}-2 \dot{m}(t) \rho_{1} \\
& -2 m(t) u_{1}, \\
\dot{e}_{2} & =a_{2} e_{3}+2 u_{2}
\end{aligned}
$$




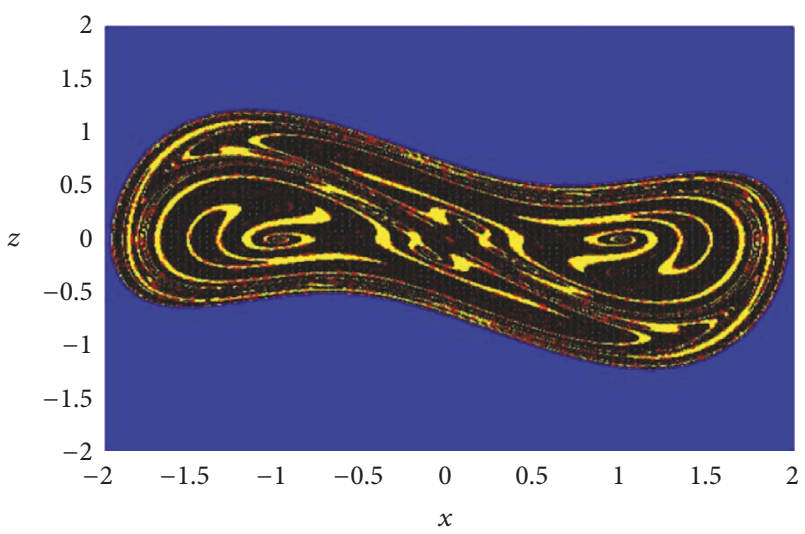

FIgURE 7: Cross-section for $x_{2}=0$ of the basins of the symmetric pair period-3 cycle (yellow), pair of period-6 (red), and the pair of chaotic attractors (black) of system (2) at $a=17.37$ and $\gamma=0.7401$. Blue zones correspond to unbounded solutions (color figure online).

$$
\begin{aligned}
\dot{e}_{n} & =\gamma_{2} e_{2}+\gamma_{3} e_{3}+\cdots+\gamma_{n} e_{n}+f\left(e_{1}, x_{1}, \rho_{1}, y_{1}, m(t)\right) \\
& +2 u_{n},
\end{aligned}
$$

where $f\left(e_{1}, x_{1}, y_{1}, \rho_{1}, m(t)\right)=p\left(x_{1}\right)+p\left(y_{1}\right)-2 p\left(\rho_{1}\right)$.

Let us choose the controllers in the following form:

$$
\begin{aligned}
& u_{1}=\frac{1}{2 m(t)}\left(a_{1}\left(x_{2}+y_{2}\right)-2 a_{1} m(t) \rho_{2}-2 \dot{m}(t) \rho_{1}\right. \\
& \left.\quad+\xi\left|e_{1}\right|^{\gamma_{0}} \operatorname{sign}\left(e_{1}\right)\right) \\
& u_{n}=-\frac{1}{2}\left(f\left(0, x_{1}, \rho_{1}, y_{1}, m(t)\right)\right) \\
& u_{2}=0 \\
& u_{(n-1)}=0 .
\end{aligned}
$$

Theorem 4. For the given scaling function matrix $m(t)$, the projective function synchronization in relay coupled oscillators between outer $X$ and outer $Y$ will occur under the relay (6) and the control law (10a), (10b), and (10c).

Proof. In order to prove the stability of the scheme, let us divide the errors dynamics (9) into two subsystems.

\section{Subsystem 1}

$$
\begin{aligned}
\dot{e}_{1}= & -k e_{1}+a_{1}\left(x_{2}+y_{2}\right)-2 a_{1} m(t) \rho_{2}-2 \dot{m}(t) \rho_{1} \\
& -2 m(t) u_{1} .
\end{aligned}
$$

Subsystem 2

$$
\begin{aligned}
\dot{e}_{2} & =a_{2} e_{3}+2 u_{2} \\
\vdots & \\
\dot{e}_{n} & =\gamma_{2} e_{2}+\gamma_{3} e_{3}+\cdots+\gamma_{n} e_{n}+f\left(e_{1}, x_{1}, \rho_{1}, y_{1}, m(t)\right) \\
& +2 u_{n} .
\end{aligned}
$$

The criterion based on asymptotic stability which states that "if the subsystem (11) is stable under the controller (10a), then the overall stability of the scheme is guaranteed under the controller (10b) and (10c)" was developed as necessary and sufficient condition for the synchronization of the periodic and chaotic systems [25]. On the basis of this criterion, let us seek a suitable Lyapunov function to establish the asymptotic stability of subsystems (11) and (12).

The errors of the scheme become

$$
\begin{aligned}
\dot{e}_{2} & =a_{2} e_{3} \\
\vdots & \\
\dot{e}_{n} & =\gamma_{2} e_{2}+\gamma_{3} e_{3}+\cdots+\gamma_{n} e_{n}+f\left(e_{1}, x_{1}, \rho_{1}, y_{1}, m(t)\right) \\
& -f\left(0, x_{1}, \rho_{1}, y_{1}, m(t)\right) .
\end{aligned}
$$

Construct the dynamical Lyapunov function as follows:

$$
V=\frac{1}{2} \sum_{i=2}^{n} e_{i}^{2}
$$

Then the time derivative of Lyapunov function $V$ along the trajectory of error system (13) is

$$
\begin{aligned}
& \frac{d V}{d t}=\dot{e}_{2} e_{2}+\cdots+\dot{e}_{n} e_{n}=\alpha_{i} \sum_{i=2}^{n} e_{i} e_{i+1}+\gamma_{n} e_{n}^{2} \\
& +\left[f\left(e_{1}, x_{1}, \rho_{1}, y_{1}, m(t)\right)-f\left(0, x_{1}, \rho_{1}, y_{1}, m(t)\right)\right] \\
& \quad \cdot e_{n},
\end{aligned}
$$

where $\alpha_{i}$ is a constant depending on the system parameters. Since subsystem (11) is stable and $e_{1}(t) \rightarrow 0$ then expression (15) becomes

$$
\frac{d V}{d t}=\sum_{i=2}^{n} \alpha_{i} e_{i} e_{i+1}+\gamma_{n} e_{n}^{2} \leq r^{2} \sum_{i=2}^{n} \alpha_{i}+\gamma_{n} r^{2} .
$$

The stability is guaranteed if expression (16) is negative. This is possible if $\gamma_{n}=-\sum_{i=2}^{n} \alpha_{i}$ which is consistent with a general class of jerk system $[26,27]$. The proof is complete.

Definition 5. Consider outer 1 and outer 2 described by systems (4) and (5), respectively. If there exists a constant $t_{r}=t_{r}(e(0))>0$, such that

$$
\lim _{t \rightarrow t_{r}}\|e(t)\|=0
$$

and $\|e(t)\| \equiv 0$ when $t \geq t_{r}$, then the chaos synchronization in relay coupled systems (3) and (4) is achieved in a finite time. 
Theorem 6. For any nonzero scaling function $m(t)$, the outer system (3) can synchronize the outer system (4) with relay (6) in a finite time given by

$$
t_{r}=\frac{1}{\left(1-\gamma_{0}\right) \min (k, \xi)} \ln \left(1+\left|e_{1}(0)\right|^{1-\gamma_{0}}\right) .
$$

Proof. Let us choose as a Lyapunov function candidate

$$
U=\left|e_{1}\right|
$$

The time derivative of $U$ along the trajectories of the closedloop system (8) is given by

$$
\begin{aligned}
\frac{d U}{d t} & =\dot{e}_{1} \operatorname{sign}\left(e_{1}\right) \\
& =\left[-k e_{1}-\xi\left|e_{1}\right|^{\gamma_{0}} \operatorname{sign}\left(e_{1}\right)\right] \operatorname{sign}\left(e_{1}\right) \\
& =-k\left|e_{1}\right|-\xi\left|e_{1}\right|^{\gamma_{0}} .
\end{aligned}
$$

Now, let $\eta=\min (k, \xi)$, then we obtain

$$
\frac{d U}{d t}=-\eta\left[\left|e_{1}\right|+\left|e_{1}\right|^{\gamma_{0}}\right] \leq-\eta\left[U+U^{\gamma_{0}}\right] .
$$

From (21), we can obtain

$$
t_{r}-t_{0} \leq-\frac{1}{\eta} \int_{t_{0}}^{t_{r}} \frac{U^{-\gamma_{0}}}{\left(1+U^{1-\gamma_{0}}\right)} d U
$$

If we suppose that $t_{0}=0$ and $U\left(t_{r}\right)=0$ since the synchronization is completed, hence

$$
t_{r} \leq \frac{1}{\eta\left(1-\gamma_{0}\right)} \ln \left(1+U^{1-\gamma_{0}}\left(t_{0}\right)\right)
$$

In addition $U\left(t_{0}\right)=\left|e_{1}(0)\right|$; hence

$$
t_{r} \leq \frac{1}{\eta\left(1-\gamma_{0}\right)} \ln \left(1+\left|e_{1}(0)\right|^{1-\gamma_{0}}\right)
$$

This proof is complete.

Remark 7. The control objective is stated in the mathematical form in (10a), (10b), and (10c). One advantage of this type of controller is that it can be easily constructed through time varying resistors, capacitors, or operational amplifiers and their combinations or using a digital signal processor together with the appropriate converters. The proposed work uses a reduced number of control signals and parameters.

3.2. Numerical Results. In this section, a numerical example and its simulations are presented to demonstrate the effectiveness of the proposed scheme. To illustrate our method, we choose the same circuit where the dynamical analysis is

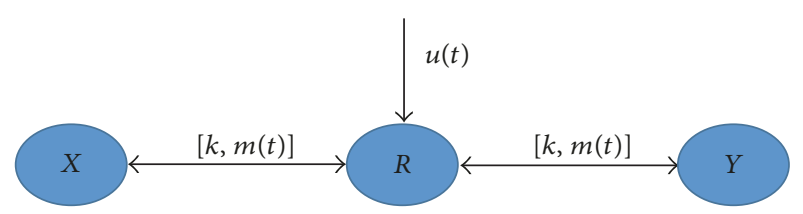

FIGURE 8: Schematic representation of the relay mechanism. Oscillators $X$ and $Y$ are coupled bidirectionally to oscillator $R$ with coupling strength $k$.

described previously in this work. The dynamic equations of outers is as follows, respectively, for $X(t)$ and $Y(t)$ :

$$
\begin{aligned}
(X) \quad \dot{x}_{1} & =x_{2}-k\left(x_{1}-m(t) \rho_{1}\right), \\
\dot{x}_{2} & =a x_{3}, \\
\dot{x}_{3} & =-b x_{2}-x_{3}+p\left(x_{1}\right), \\
(Y) \quad \dot{y}_{1} & =y_{2}-k\left(y_{1}-m(t) \rho_{1}\right), \\
\dot{y}_{2} & =a y_{3}, \\
\dot{y}_{3} & =-b y_{2}-y_{3}+p\left(y_{1}\right),
\end{aligned}
$$

where $a$ and $b$ are the positive constants and $p\left(\bullet_{1}\right)$ is the polynomial smooth function.

For instance, the system is chaotic for the parameters $a=10, \gamma=0.725, p\left(\theta_{1}\right)=\theta_{1}-\theta_{1}^{3}$ for any variable $\theta_{1}$. $m(t)$ is the scaling function and $k$ is the coupling strength. The impact of $k$ and $m(t)$ on the dynamical behaviors of the entire coupled systems will be investigated later. We set the following parameters: $a=18.1$ and $\gamma=0.625$. From (10a), (10b), and (10c) the controllers take the form

$$
\begin{aligned}
u_{1} & =\frac{1}{2 m(t)}\left(\left(x_{2}+y_{2}\right)-2 m(t) \rho_{2}-2 \dot{m}(t) \rho_{1}\right. \\
& \left.+\xi\left|e_{1}\right|^{\gamma} \operatorname{sign}\left(e_{1}\right)\right), \\
u_{2} & =\frac{1}{2}\left(2(m(t)-1) \rho_{1}+\left(2-8 m^{3}(t)\right) \rho_{1}^{3}\right. \\
& \left.+6 m(t) x_{1} y_{1} \rho_{1}\right) .
\end{aligned}
$$

Let us consider the following parameters: $\xi=0.01, k=2$ and $\eta=0.01$. The scaling function $m(t)$ is expressed as $m(t)=e_{0}+0.1 \sin (\pi \phi t)$ with $e_{0}$ and $\phi$ the positive constants. Results of our "relay" synchronization in a system of three circadian oscillators are shown in Figure 8. The repressors from the three subsystems are labeled $X, R$, and $Y$, respectively. Although each of the oscillators is identical, the initial conditions of the three circuits are different. Whatever the situation of the systems, Wagemakers et al. showed that outer systems can synchronize even if the relay unit has different architecture [16]. There is phase synchrony between the repressor output from oscillators $X$ and $Y$. If we set the following values of $e_{0}$ and $\phi$ to 0.75 and 0.7 , respectively, the attractors of different systems are provided in Figure 9. The diagrams in blue and black represent the outer 1 and outer 2, respectively. The similarity of these graphs is complete. The 


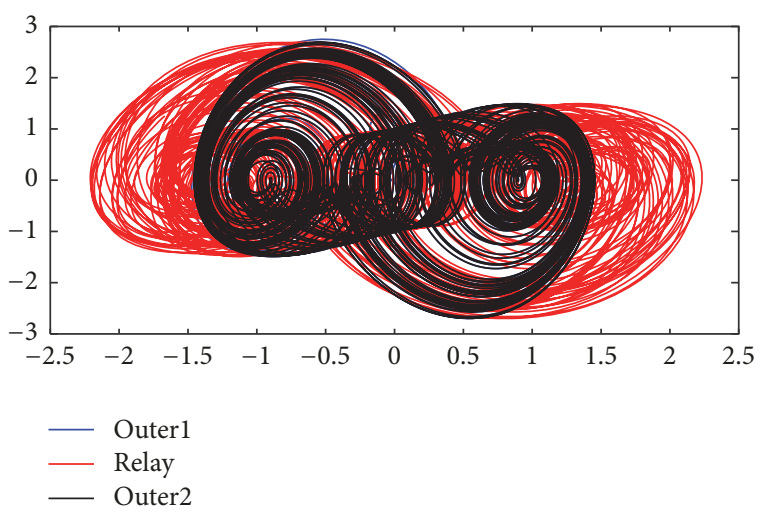

Figure 9: Chaotic behavior for $e_{0}=0.75 \phi=0.7$ (blue and black attractors correspond to outer 1 and outer 2 , and the third in red represents the relay).
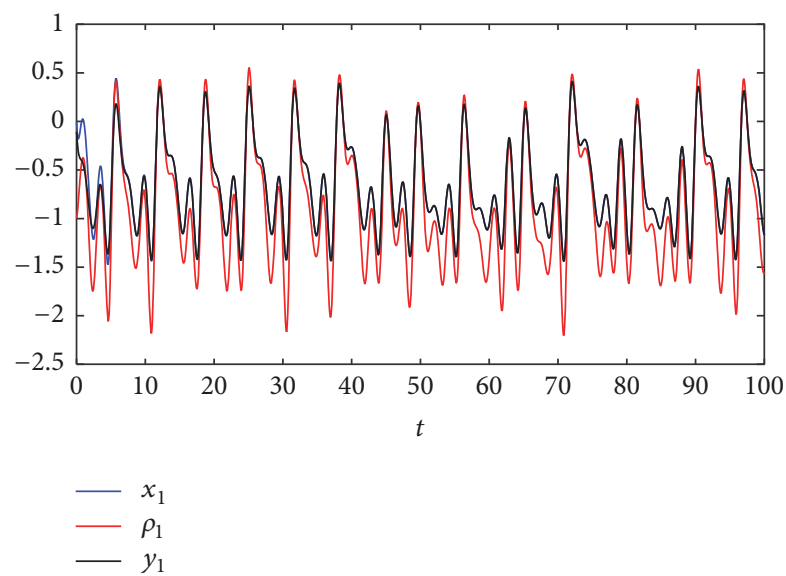

FIgURE 10: Cluster synchronization.

diagram in red displays the relay. The chaotic structure of the relay system renders its manipulation bulky and difficult for an attacker in the practical situations.

One of the major collective coherent behaviors in ensembles of identical and nonidentical chaotic elements is global and cluster synchronization. For complete synchronization we focus on the existence and stability of unique unconditional clusters whose rise does not depend on the origin of the outers. The cluster synchronization is established in relation with the difference between the relay and the outers. Figure 10 presents the diagrams showing the cluster synchronization. Figure 11 displays the global errors of the system given by (7). It is important to observe that the stabilization of the one state leads to other states of the scheme which is consistent with our theory analysis. The errors of synchronization between the outers are provided in Figure 12. From these graphs, we remark that the relay circuit allows finite synchronization and in a short time.

Remark 8. A typical assumption made by most chaotic cryptosystems' designers is that the system's parameters play the role of the key which is not seen as efficient in many secure schemes. In this paper we assume that $m(t)$ plays the role of

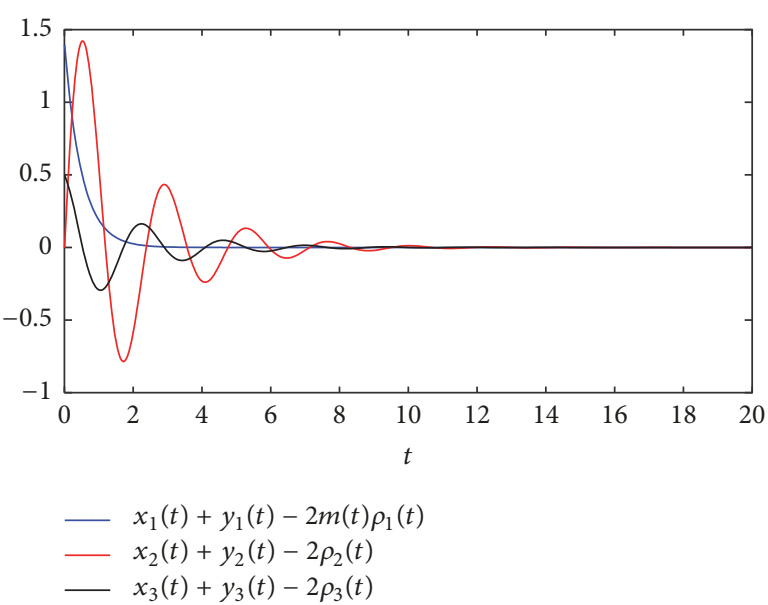

FIgURE 11: The global errors state of synchronization.

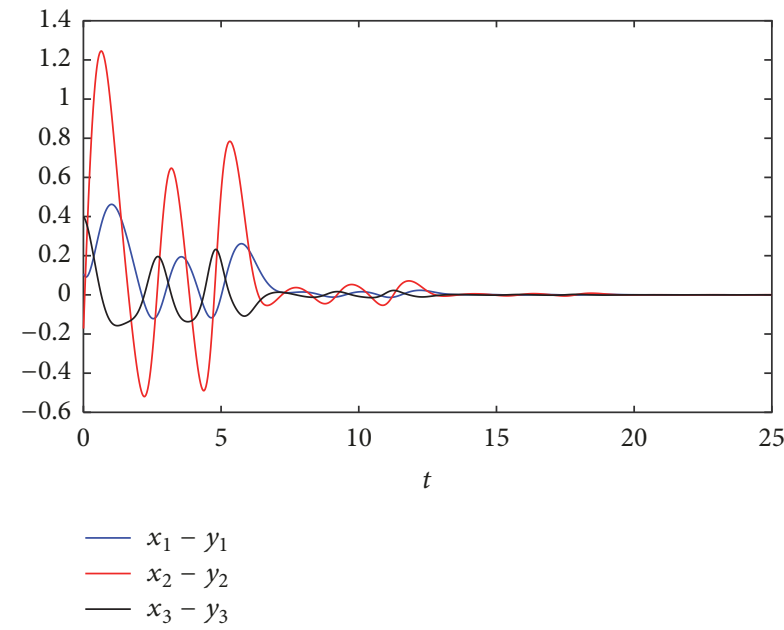

FIGURE 12: Synchronization errors between the outers.

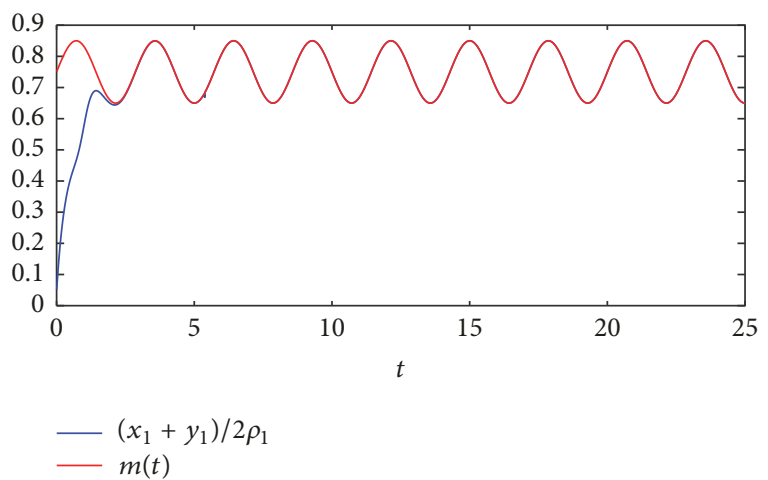

FIGURE 13: Original function (red); retrieved function (blue) for $a=$ 18.1 and $\gamma=0.625$.

the key. From (7) we can derive $e_{1}(t)=x_{1}+y_{1}-2 m(t) \rho_{1} \rightarrow 0$ as $t \rightarrow t_{r}$; that is, $\left(x_{1}+y_{1}\right) / 2 \rho_{1}$ can recover the message signal $m(t)$. Figure 13 depicts the function $m(t)$. The original is plotted in red and the same function is recovered after a short transient behavior. 


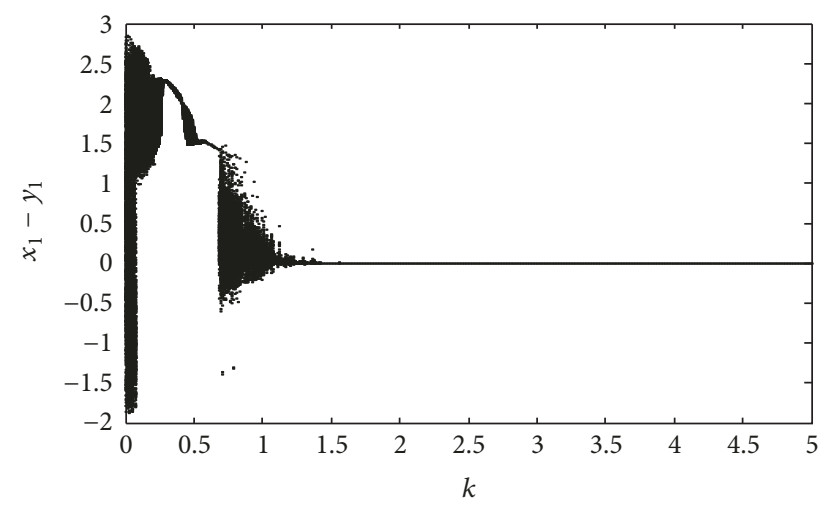

FIGURE 14: Bifurcation diagram with $k \in[0 ; 5]$.

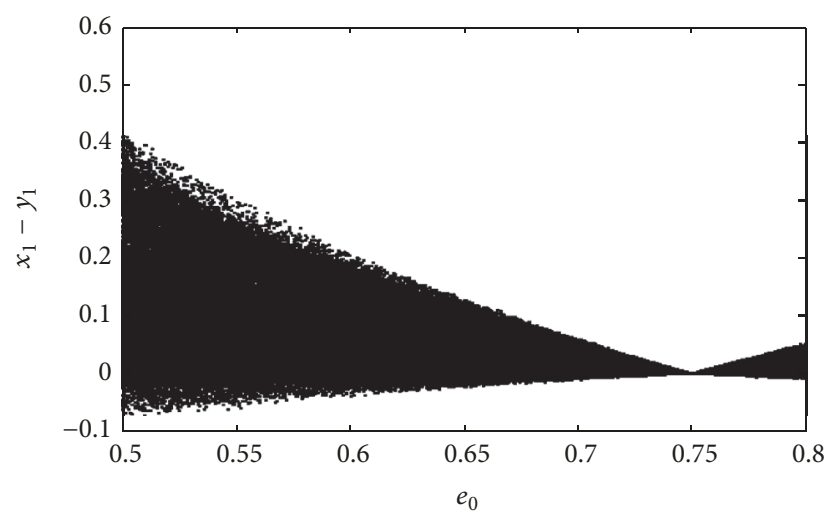

FIgURE 15: Bifurcation diagram $e_{o} \in[0.5,0.8]$.

3.3. Scaling Function and "Relay" Synchronization. The scheme described above depicts many interesting features. It is possible to analyze a number of situations that are likely to arise when the parameters of the scaling function are monitored. For instance, when chaos synchronization is applied in engineering applications such as in secure communications, the chaos-based synchronization scheme is rather sensitive to attacks. It is necessary to analyze the interactions of the scaling function parameters which is naturally considered as a key and the coupling strength $k v i s-\grave{a}-v i s$ of the scheme. For small values of the coupling parameter $k$, the chaotic regime is asynchronous; that is, $X \neq Y$. Transition from asynchronous to synchronous generation occurs through the intermittency of "bubbling" type $[28,29]$. Figure 14 shows that the variation of the control parameters allows different behaviors which are periodic or chaotic behavior. One can see that with the increase in $k$, we move towards the region of periodic synchronous generation. In this context, the optimal value of the coupling which allows the synchronization between the outers is about 1.45. If we vary the values of $e_{0}$ and $\phi$ between the intervals [0.5 0.7] and [0,5], respectively, the bifurcation diagrams given on Figures 15 and 16 show a consistent expectation of the function projective synchronization working [30]. One can see that initially the systems are asynchronous. After a while, the symmetry breaking bifurcation occurs when $e_{0}=e_{\text {opt }}$. With an increase in $e_{0}$

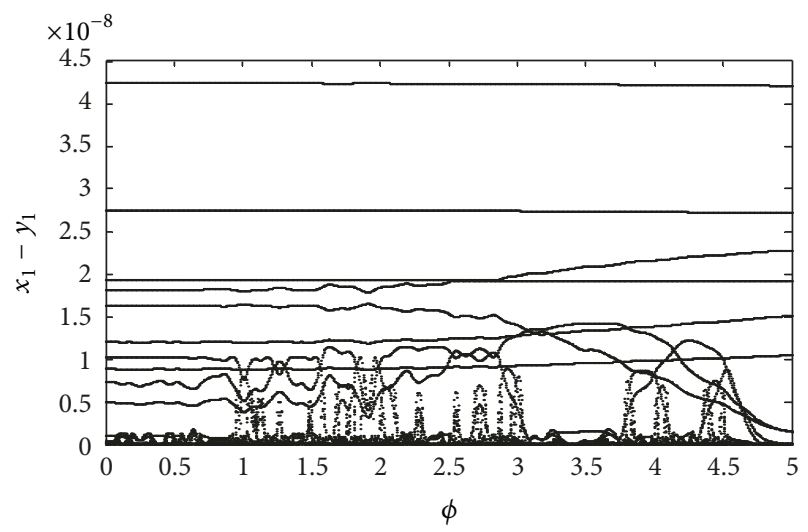

FIGURE 16: Bifurcation diagrams with $\phi \in[0,5]$.

self-modulation and transition to chaos take place. The errors system remains practically weak when the constant $\phi$ varies. Note that the knowledge of a set of parameters $\left(k, e_{\mathrm{opt}}, \phi\right)$ for unauthorized agent remains a hard test. Their determination is a requirement to detect the synchronization zone which is an excellent agreement with our theoretical predictions.

\section{Conclusions and Remarks}

In this unexplored regime of the jerk system, new regions of multistability have been found. The complex dynamics of chaotic motions are reported by means of Lyapunov exponent spectrum. By varying the initial conditions, we are also able to detect six coexisting stable attractors and three equilibrium points attractors instead of four encountered in the literature. And on the other hand, we have demonstrated that relay synchronization can be associated with function projective synchronization between the relay unit and the synchronized systems. The mediating role of FPS implies the existence of an invertible function that links the dynamics of the relay system with those of the systems to be synchronized. The key role of FPS is demonstrated by analyzing the bifurcation diagram of the whole system with respect to the parameters of the scaling function $m(t)$. Therefore, our results link the emergence of relay synchronization in instantaneously coupled chaotic systems with the existence of FPS with the relay system and open the possibility of using relay units for secure communications [31]. Comparing our results to those found in the literature [13-21], the isospike diagrams show the overall behavior of the system and the relay unit associated with the unpredictability of the scaling function appears as a fundamental for the feasibility of the reliable secure communications.

\section{Conflicts of Interest}

The authors declare that they have no conflicts of interest.

\section{References}

[1] C. Li and J. C. Sprott, "Multistability in the Lorenz system: a broken butterfly," International Journal of Bifurcation and Chaos, vol. 24, no. 10, article 1450131, 2014. 
[2] J. Kengne, Z. N. Tabekoueng, V. K. Tamba, and A. N. Negou, "Periodicity, chaos, and multiple attractors in a memristorbased Shinriki's circuit," Chaos: An Interdisciplinary Journal of Nonlinear Science, vol. 25, no. 10, article 103126, 2015.

[3] C. Hens, S. K. Dana, and U. Feudel, "Extreme multistability: attractor manipulation and robustness," Chaos: An Interdisciplinary Journal of Nonlinear Science, vol. 25, no. 5, article 053112, 2015.

[4] G. M. Maggio, O. De Feo, and M. P. Kennedy, "Nonlinear analysis of the Colpitts oscillator and applications to design," IEEE Transactions on Circuits and Systems I: Fundamental Theory and Applications, vol. 46, no. 9, pp. 1118-1130, 1999.

[5] C. Masoller, "Coexistence of attractors in a laser diode with optical feedback from a large external cavity," Physical Review A: Atomic, Molecular and Optical Physics, vol. 50, no. 3, pp. 25692578, 1994.

[6] J. M. Cushing, Henson, and S. M. Blackburn, "Multiple mixed attractors in a competition model," Journal of Biological Dynamics, vol. 1, pp. 347-362, 2007.

[7] C. Li and J. C. Sprott, "Coexisting hidden attractors in a 4-D simplified lorenz system," International Journal of Bifurcation and Chaos, vol. 24, no. 3, article 1450034, 2014.

[8] S. Rajasekar and M. Lakshmanan, "Multiple attractors and their basins of attraction of a long Josephson junction oscillator," Physics Letters A, vol. 147, no. 5-6, pp. 264-268, 1990.

[9] A. Massoudi, M. G. Mahjani, and M. Jafarian, "Multiple attractors in Koper-Gaspard model of electrochemical periodic and chaotic oscillations," Journal of Electroanalytical Chemistry, vol. 647, no. 1, pp. 74-86, 2010.

[10] M. Dutta, H. E. Nusse, J. A. Yorke, and G. Yuan, "Miltiple Attractor Bifurcations: A Source of Unpredictability in Piecewise Smooth Systems," Physical Review Letters, vol. 83, no. 21, pp. 4281-4284, 1999.

[11] S. Banerjee, P. Ranjan, and C. Grebogi, "Bifurcations in twodimensional piecewise smooth maps-theory and applications in switching circuits," IEEE Transactions on Circuits and Systems I: Fundamental Theory and Applications, vol. 47, no. 5, pp. 633643, 2000.

[12] B. C. Bao, H. Bao, N. Wang, M. Chen, and Q. Xu, "Hidden extreme multistability in memristive hyperchaotic system," Chaos, Solitons \& Fractals, vol. 94, pp. 102-111, 2017.

[13] J. Kengne, Z. T. Njitacke, and H. B. Fotsin, "Dynamical analysis of a simple autonomous jerk system with multiple attractors," Nonlinear Dynamics, vol. 83, pp. 751-765, 2016.

[14] A. R. Elsonbaty and A. M. El-Sayed, "Further nonlinear dynamical analysis of simple jerk system with multiple attractors," Nonlinear Dynamics, vol. 87, no. 2, pp. 1169-1186, 2017.

[15] S. Boccaletti, J. Kurths, G. Osipov, D. L. Valladares, and C. S. Zhou, "The synchronization of chaotic systems," Physics Reports, vol. 366, no. 1-2, pp. 1-101, 2002.

[16] A. Wagemakers, J. M. Buldú, and M. A. F. Sanjuán, "Isochronous synchronization in mutually coupled chaotic circuits," Chaos: An Interdisciplinary Journal of Nonlinear Science, vol. 17, no. 2, article 023128, 2007.

[17] A. Sharma, P. R. Sharma, and M. D. Shrimali, "Amplitude death in nonlinear oscillators with indirect coupling," Physics Letters A, vol. 376, no. 18, pp. 1562-1566, 2012.

[18] A. Sharma, M. Dev Shrimali, and S. Kumar Dana, "Phaseflip transition in nonlinear oscillators coupled by dynamic environment," Chaos: An Interdisciplinary Journal of Nonlinear Science, vol. 22, no. 2, article 023147, 2012.
[19] V. Raul, L. G. Leonardo, R. M. Claudio et al., "Dynamical relaying can yield zero time lag neuronal synchrony despite long conduction delays," Proceedings of the National Acadamy of Sciences of the United States of America, vol. 105, no. 44, pp. 17157-17162, 2008.

[20] A. Wagemakers, J. M. Buldú, and M. A. Sanjuán, "Experimental demonstration of bidirectional chaotic communication by means of isochronal synchronization," EPL (Europhysics Letters), vol. 81, no. 4, article 40005, 2008.

[21] B. Nana and P. Woafo, "Chaotic masking of communication in an emitter-relay-receiver electronic setup," Nonlinear Dynamics, vol. 82, no. 1-2, pp. 899-908, 2015.

[22] R. Gutiérrez, R. Sevilla-Escoboza, P. Piedrahita et al., "Generalized synchronization in relay systems with instantaneous coupling," Physical Review E: Statistical, Nonlinear, and Soft Matter Physics, vol. 88, no. 5, article 052908, 2013.

[23] S. T. Kammogne and H. B. Fotsin, "Adaptive control for modified projective synchronization-based approach for estimating all parameters of a class of uncertain systems: case of modified colpitts oscillators," Journal of Chaos, vol. 2014, Article ID 659647, 13 pages, 2014.

[24] D. F. Oliveira and E. D. Leonel, "Some dynamical properties of a classical dissipative bouncing ball model with two nonlinearities," Physica A: Statistical Mechanics and its Applications, vol. 392, no. 8, pp. 1762-1769, 2013.

[25] R. A. Freeman and P. V. Kokotovic, Robust Nonlinear Control Design, Birkhäauser, Boston, Mass, USA, 1996.

[26] J. G. Freire and J. A. C. Gallas, "Cyclic organization of stable periodic and chaotic pulsations in Hartley's oscillator," Chaos, Solitons \& Fractals, vol. 59, pp. 129-134, 2014.

[27] J. C. Sprott, Elegant Chaos: Algebraically Simple Chaotic Flows, World Scientific Publishing, Toh Tuck Link, Singapore, 2010.

[28] T. Divoux, E. Bertin, V. Vidal, and J.-C. Géminard, "Intermittent outgassing through a non-Newtonian fluid," Physical Review E: Statistical, Nonlinear, and Soft Matter Physics, vol. 79, no. 5, article 056204, 2009.

[29] R. Lakkaraju, F. Toschi, and D. Lohse, "Bubbling reduces intermittency in turbulent thermal convection," Journal of Fluid Mechanics, vol. 745, pp. 1-24, 2014.

[30] A. Boulkroune, A. Bouzeriba, and T. Bouden, "Fuzzy generalized projective synchronization of incommensurate fractionalorder chaotic systems," Neurocomputing, vol. 173, pp. 606-614, 2016.

[31] W. Kinzel, A. Englert, and I. Kanter, "On chaos synchronization and secure communication," Philosophical Transactions. Series A, Mathematical, Physical, and Engineering Sciences, vol. 368, pp. 379-389, 2010. 


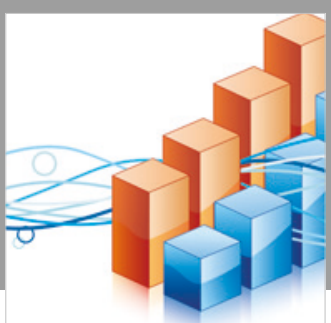

Advances in

Operations Research

\section{-n-m}
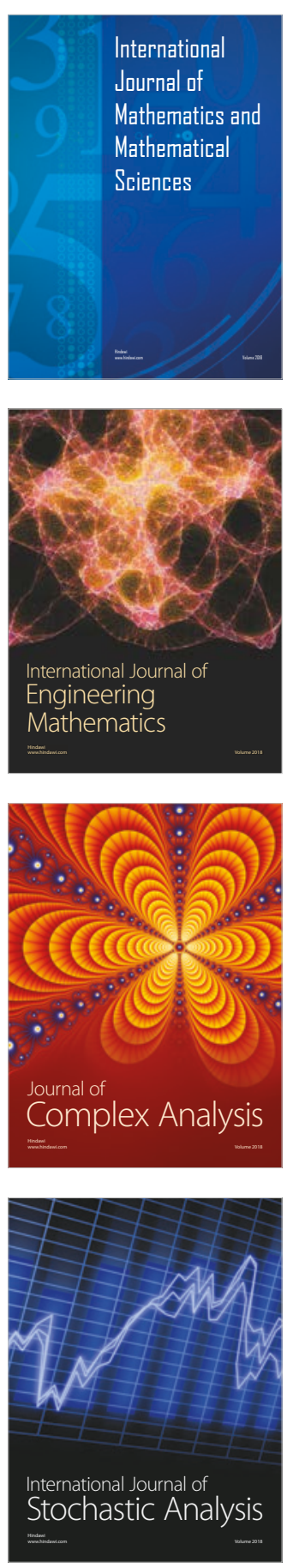
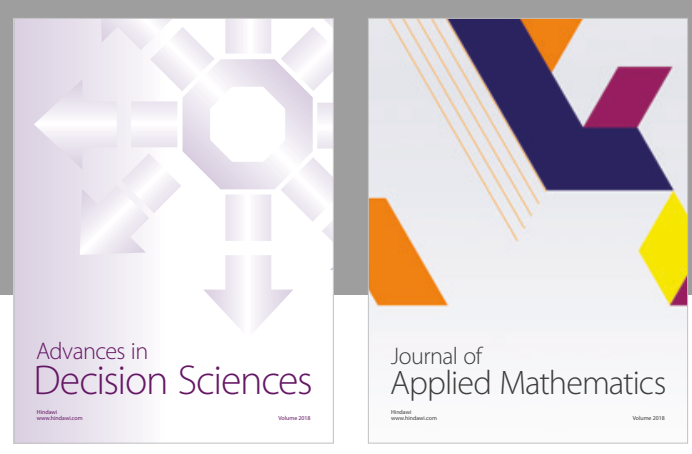

Journal of

Applied Mathematics
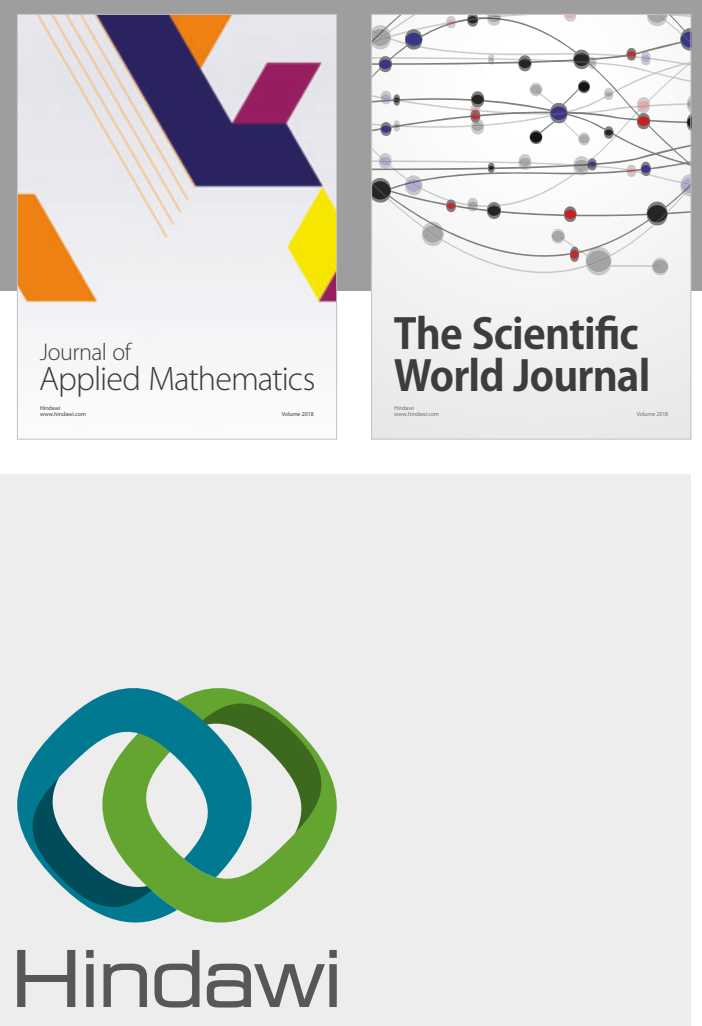

Submit your manuscripts at

www.hindawi.com

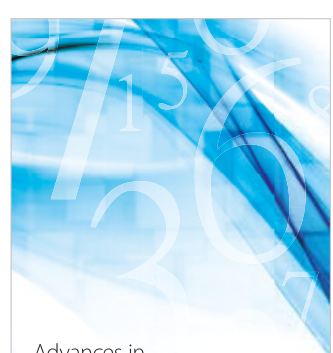

Advances in
Numerical Analysis
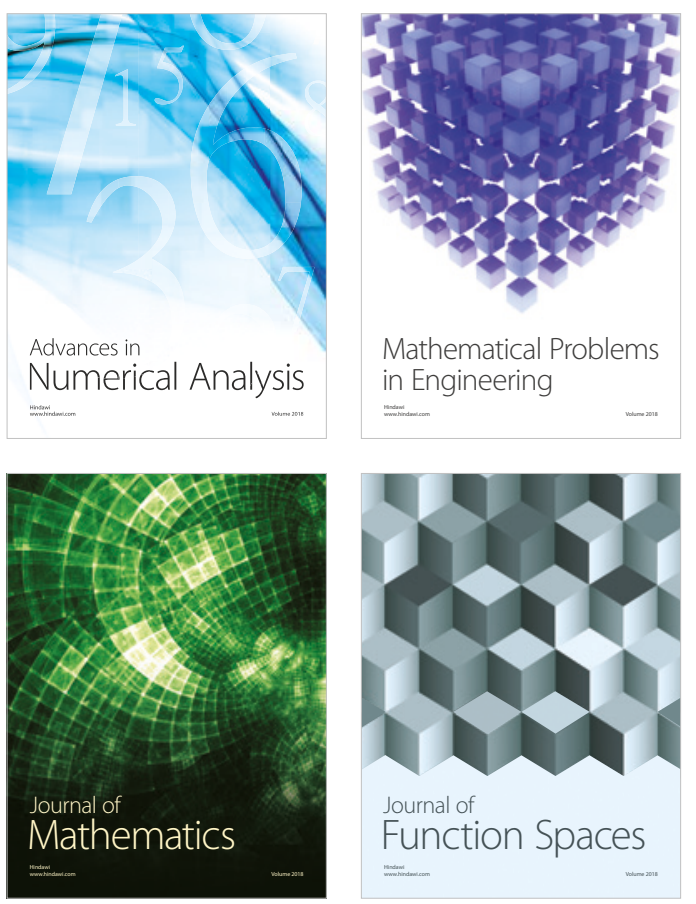

Mathematical Problems in Engineering

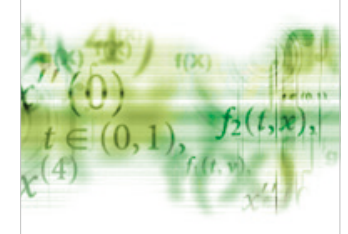

International Journal of

Differential Equations

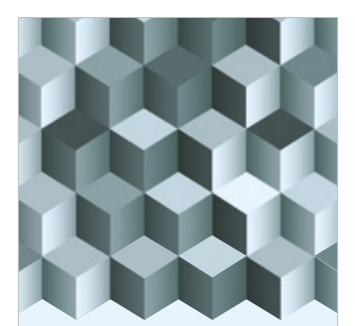

Journal of

Function Spaces
The Scientific

World Journal

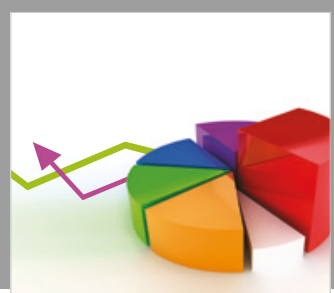

Journal of

Probability and Statistics
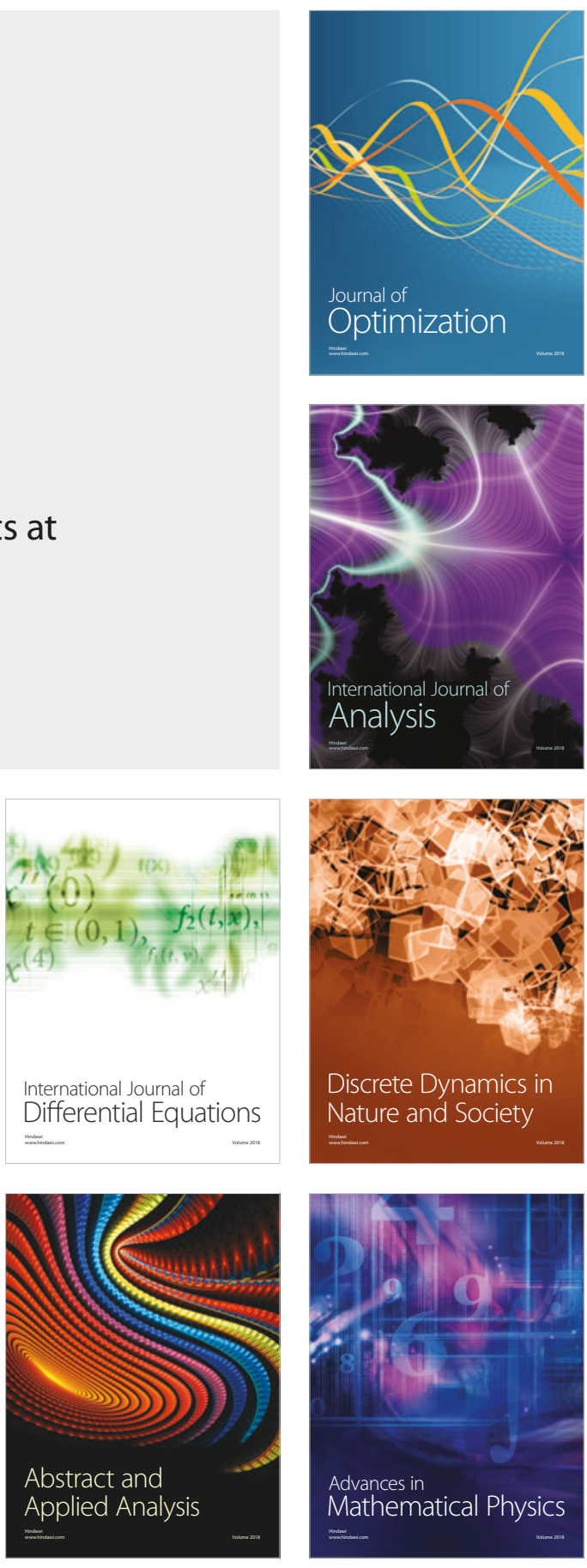